livraisons

d'Histoire

de l'Architecture

\section{Livraisons de l'histoire de l'architecture}

$40 \mid 2020$

À propos des princes de Conti

\title{
Les derniers princes de Bourbon Conti et la sculpture : collectionneurs et commanditaires
}

Les derniers princes de Bourbon Conti et la sculpture : collectionneurs et commanditaires

The last princes of Bourbon Conti and sculpture. Collectors and Sponsors Die letzten Fürsten von Bourbon-Conti und die Bildhauerei. Sammler und Auftraggeber

\section{Frédéric Chappey}

\section{OpenEdition} Journals

Édition électronique

URL : http://journals.openedition.org//ha/1268

DOI : $10.4000 /$ lha. 1268

ISSN : 1960-5994

Éditeur

Association Livraisons d'histoire de l'architecture - LHA

Édition imprimée

Date de publication : 3 décembre 2020

Pagination : 23-32

ISSN : 1627-4970

\section{Référence électronique}

Frédéric Chappey, «Les derniers princes de Bourbon Conti et la sculpture : collectionneurs et commanditaires », Livraisons de l'histoire de l'architecture [En ligne], 40 | 2020, mis en ligne le 28 décembre 2020, consulté le 24 mars 2021. URL : http://journals.openedition.org//ha/1268 ; DOI : https://doi.org/10.4000//ha.1268 
Par Frédéric CHAPPEY

\section{LES DERNIERS PRINCES DE BOURBON CONTI ET LA SCULPTURE : COLLECTIONNEURS ET COMMANDITAIRES}

Nombreux sont les ouvrages anciens et les écrits récents se rapportant au goût immodéré pour la peinture dont a témoigné Louis-François de Bourbon Conti (1717-1776) durant sa longue vie de prince du sang et dont témoignent les deux ventes, posthumes et pléthoriques, de ses collections, en 1777 et 1779 . Plus rares sont ceux qui se sont intéressés au goût de ce dernier et de son fils Louis-FrançoisJoseph de Bourbon Conti (1734-1814) pour la sculpture, soit en tant que collectionneurs, soit en tant que commanditaires afin de décorer d'une part, leurs demeures princières et au premier chef, la plus célèbre d'entre elles, leur bien-aimé château de L'Isle-Adam, situé près d'Auvers-sur-Oise, et d'autre part, leur ambitieuse chapelle funéraire adamoise. Sans doute n'était-il pas inopportun de revisiter ce sujet d'étude particulier dans le cadre du contexte plus général de l'histoire du goût de la haute aristocratie et des élites culturelles pour la sculpture à la fin de l'Ancien Régime ${ }^{1}$.

\section{Les ventes Conti de 1777 et 1779}

Le catalogue de la vente du 8 avril au 8 juin 1777 ordonnée par le nouveau prince de Conti pour se désengager des dettes considérables contractées par son père Louis-François de Bourbon Conti, nous renseigne utilement sur l'intérêt de ce dernier pour la sculpture ancienne mais également pour certains sculpteurs contemporains de son époque ${ }^{2}$. Plus de 130 œuvres sculptées, sans compter plus d'un millier de médailles, sont proposées à la vente et seront adjugées pour plus de 28000 livres, quand les 1100 peintures et les quelques 400 dessins seront, eux, cédés pour un total d'environ 1100000 livres. Cependant, il est vraisemblable que d'autres sculptures encore, aient pu volontairement être conservées par le fils,

1. Frédéric Bussmann, Un Prince collectionneur. Louis-François de Bourbon Conti et ses collections au palais du Temple à Paris, Paris, éd. de la Maison des Sciences de l'homme et du Centre allemand d'histoire de l'art, 2012, 566 pages; G. Scherf, "Collections et collectionneurs de sculptures modernes. Un nouveau champ d'études ", L'Art et les Normes sociales au XVIII e siècle, Paris, 2001, Éd. de la Maison des sciences de l'Homme, p. 147-164.

2. Cat. exp. Les Trésors des princes de Bourbon Conti, L'Isle-Adam, musée d'art et d'histoire Louis Senlecq, 28 mai- $^{\text {er }}$ octobre 2000, sous la direction de Frédéric Chappey, 168 pages (avec bibliographie générale). 
Louis-François-Joseph, pour son usage personnel ou par inclinaison particulière. Une première série d'œuvres, malheureusement anonymes, est inventoriée dans le catalogue de vente. Elle est composée d'une douzaine de vases en bronze, de marbre et d'améthyste, signes caractéristiques d'une collection princière prestigieuse, qui représenteront néanmoins à eux seuls un peu plus du tiers du montant total adjugé, ainsi que d'une trentaine de bronzes à l'antique, grecs et égyptiens, de trois basreliefs en marbre et huit bas-reliefs en cire, et enfin d'une vingtaine de "bronzes modernes". Une seconde série est constituée des rares œuvres attribuées à des sculpteurs identifiés par les experts de la vente. Au sein de cette dernière série, deux catégories de sculptures peuvent être déterminées. Tout d'abord, celles relevant des siècles passés, comme deux terres cuites attribuées à Jean Goujon (vers 1510-vers 1567), mais surtout comme les 43 œuvres de François Duquesnoy (1597-1643), dit à l'époque François Flamand, qui apparaît indiscutablement comme le sculpteur le mieux représenté dans la collection princière. Puis enfin, se distingue un dernier groupe d'une trentaine d'œuvres, toutes en terre-cuite, composé de celles données à des sculpteurs contemporains du collectionneur, comme Edmé Bouchardon (1698-1762) dont le prince collectionne également les dessins, Jean-Baptiste Pigalle (1714-1785), Louis-Claude Vassé (1714-1772), Augustin Pajou (1730-1809), LouisFélix Delarue (1730-1777), Louis-Simon Boizot (1743-1809) et surtout Claude Michel, dit Clodion (1738-1814), qui lui, est l'artiste moderne le mieux représenté dans cette collection Conti avec une douzaine de sculptures. Louis-François de Bourbon Conti semble d'ailleurs avoir été l'un de ses premiers collectionneurs, sinon même, l'un de ses premiers commanditaires, comme avec les deux vases avec décor de jeux d'enfants et têtes de satyres béliers (avant 1763, Saint-Pétersbourg, musée de l'Ermitage) ou les deux reliefs du Sacrifice à l'Amour et de la Marchande d'amours (Salon de 1773, Paris, vente Sotheby's, 19-20 décembre 2017, n $^{\text {os }} 293$ et 294).

Curieusement, ne se retrouvent pas au sein de cette liste d'artistes, les noms des sculpteurs Jean-Guillaume Moitte (1746-1810) et Pierre Mérard (c. 1740-1800), auxquels Louis-François de Bourbon Conti passera pourtant d'importantes commandes de sculptures décoratives pour son cher château de L'Isle-Adam. Mais nous savons néanmoins aujourd'hui que plusieurs œuvres attribuées à Clodion par les " experts" de la vente, ont bien Mérard pour auteurs, comme La Fuite en Égypte (relief, coll. part.) et deux autres reliefs circulaires, redonnés justement à ce sculpteur dans une vente parisienne, quelques années plus tard.

Demeure le goût personnel et plutôt rare à son époque, de ce prince pour l'art moderne de son temps qui lui fait collectionner des sculpteurs contemporains ou de sa génération (ill. 1). La collection Conti en sculpture était ainsi constituée principalement d'une magistrale série d'une quarantaine d'œuvres de Duquesnoy se répartissant entre une trentaine de terres cuites, huit bronzes, deux reliefs en ivoire et deux " bois ", représentant majoritairement des enfants, le sujet favori de l'artiste et de son collectionneur, mais également un faune, un buste de la Vierge et deux têtes de femmes à l'antique (cf. Enfant qui se penche en avant et du côté gauche, statuette en terre cuite, Écouen, musée national de la Renaissance ou Enfant couché 


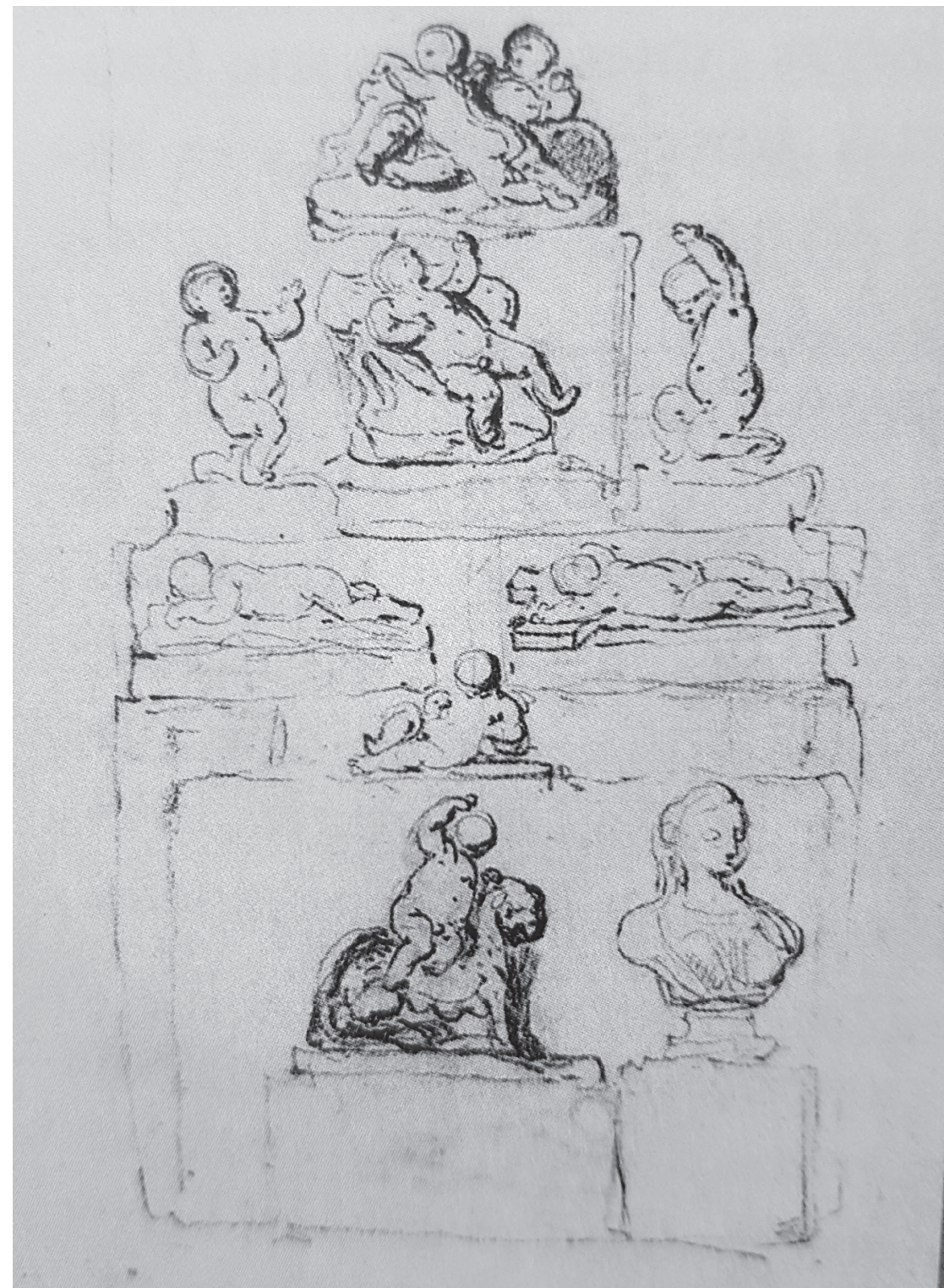

Ill. 1 : Gabriel de Saint-Aubin, Croquis au graphite d'après statuettes en terre cuite attribuées à François Duquesnoy, Augustin Pajou, Jean-Baptiste-Ignace Broche ou Louis-Félix Delarue, catalogue de la vente Conti de 1777, Paris, BNF. (C) Cl. de l'auteur.

sur le dos, une jambe tendue, l'autre repliée, une main contre la joue, l'autre bras le long du corps (Enfant Jésus endormi sur la Croix), statuette en terre cuite, Anvers, museum Mayer van den Bergh). Le goût avéré de Louis-François pour les icono- 
graphies arcadiennes et plus spécialement bacchique et enfantine, peut probablement être mis en rapport avec les tendances personnelles, libertines et frivoles de ce prince, pour des sujets aimables et plaisants à regarder, mais il s'inscrit indéniablement aussi dans le cadre plus général de l'histoire du goût des collectionneurs de sculptures de son époque, à partir de la seconde moitié du XVIII ${ }^{\mathrm{e}}$ siècle, comme PierreJean Mariette (1694-1774), Ange-Laurent La Live de Jully (1725-1779) ou encore Jacques-Onésyme Bergeret de Grandcourt (1715-1785), ce dernier, voisin des Conti à L'Isle-Adam. Tous ont à cœur de posséder de petites sculptures décoratives d'intérieur, dites sculptures de cabinet, et particulièrement des statuettes en terre cuite, qu'elles soient abouties ou esquissées, afin d'y sentir, comme disait La Live de July "le feu et le véritable talent de l'artiste " ${ }^{3}$. C'est d'ailleurs à l'occasion des ventes de certaines de ces dernières prestigieuses collections (Mariette en 1768 et La Live de July en 1770) que le prince de Conti acquiert des sculptures pour sa propre collection, quand il n'achète pas directement aux artistes eux-mêmes, comme pour Clodion. Mais contrairement aux collections royales et à d'autres collections princières comme celles des Orléans, les collections Conti, constituées assez tardivement dans la vie du prince, à partir des années 1760 , ne se visitent pas et semblent être réservées à la jouissance particulière du prince et de ses proches.

Une seconde vente Conti fut organisée à Paris par le fils du collectionneur compulsif, le 15 mars 1779 , afin de remettre en vente quelques 300 œuvres issues principalement de la première vente et non encore réglées financièrement. Le produit de cette vente atteignit la somme totale de 108000 livres. Elle était encore constituée d'une trentaine de sculptures attribuées à Duquesnoy, Clodion ou Delarue, mais également à Pierre Lepautre (1659-1744) ou à André Brenet (c. 1734- c. 1792).

\section{Le décor sculpté du château et des écuries de L'Isle-Adam}

Non content d'être un collectionneur, le prince Louis-François-Joseph fut également, entre 1778 et 1783 , un commanditaire remarquable de décors sculptés architecturaux faisant partie intégrante de l'ornementation des façades de leur château de L'Isle-Adam, près de Pontoise, et de ses belles écuries, malheureusement tous bâtiments aujourd'hui disparus, après leur destruction sous le Premier Empire. En ce qui concerne le château lui-même, imposant parallélépipède situé spectaculairement sur un promontoire bâti sur une île au milieu de l'Oise, ce prince demande à son architecte particulier Jean-Baptiste André (1735/37-après 1809) de faire décorer les deux façades, peut-être en partie par Mérard auteur probable des groupes sculptés des attiques, mais aussi par Moitte, dont ce sont sans doute parmi les premières commandes de sa carrière personnelle, et surtout par les sculpteurs ornemanistes Bastier et Jean-Joseph Lemaire, par d'ambitieux reliefs sculptés et des statues devant rappeler les hauts faits et gestes militaires des princes de Conti successifs.

3. G. Scherf, "La terre est l'affaire du génie ". "Amateurs et collections de terres cuites ", cat. exp. L'Esprit créateur. De Pigalle à Canova. Terres cuites européennes 1740-1840, Paris, musée du Louvre, New York, Stockholm, éd. RMN, 2003-2004, p. 16-21 (avec bibliographie générale de G. Scherf). 


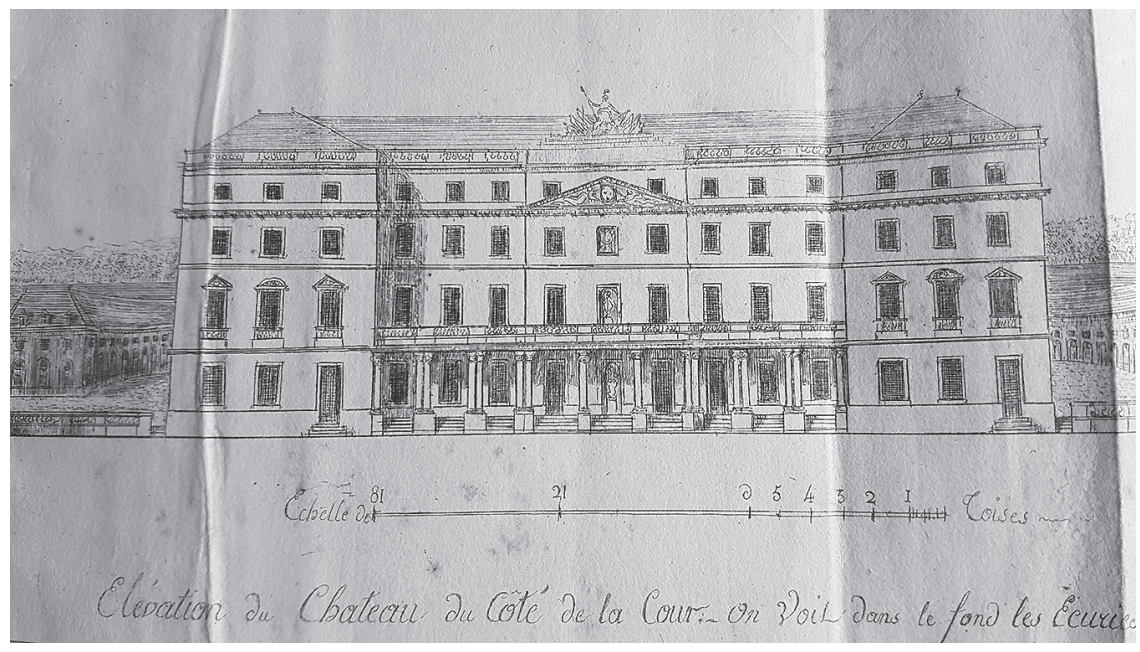

Ill. 2 : Façade donnant sur la cour d'honneur du château de L'Isle-Adam, planche gravée, Jean-Baptiste André, Chronologie historique des différents propriétaires des domaines de L'Isle-Adam (...) tracée en 1807 par M. André, ancien Architecte expert des bâtiments, avec 19 gravures, 1809. (C) Cl. de l'auteur.

Autant que nous puissions interpréter les quelques rares dessins et gravures de l'époque, sont ainsi sculptés en relief sur la façade de la cour d'honneur, de grandes armoiries des Conti encadrées de drapeaux et placées en-dessous d'un fronton en relief décoré de deux figures couchées de la Renommée retenant à nouveau les armes des Conti, fronton, lui-même surmonté d'un long relief exécuté par Moitte et représentant un combat de cavalerie, servant lui-même aussi, de haut piédestal à un groupe sculpté figurant une allégorie de la Victoire casquée et encadrée de trophées et de drapeaux. D'autres sculptures encore, semblent avoir été disposées également dans des niches au centre de l'avant-corps central, sur les trois premiers niveaux (ill. 2).

Sur la façade donnant sur les écuries, le décor sculpté en pierre consiste d'une part, en un gigantesque arbre à ramures s'étageant sur trois niveaux, transcription en pierre de la devise latine de Louis-François, "ex agitas stipit ventos" (tu as beau agiter les feuilles, tu ne pourras ébranler l'arbre), adoptée en réaction contre l'ostracisme dont il fit l'objet de la part de la marquise de Pompadour à partir de $1757^{4}$, arbre lui-même surmonté d'un large fronton cintré surbaissé et décoré d'un relief à sujet apparemment guerrier, lui-même encore surmonté d'un groupe constitué des armes des Bourbons Conti couronnées et encadrées à nouveau de deux figures allégoriques de la Renommée, assises et sonnant de leurs trompettes. Le perron de l'escalier menant à l'entrée principale sur cour du château, devait être encadré par deux sphinx, sculptés également par Jean-Guillaume Moitte (ill. 3).

4. Je remercie Jean-Michel Leniaud et surtout Christophe Morin, maître de conférences en histoire de l'art de l'université de Tours, de m'avoir signalé cette interprétation. 


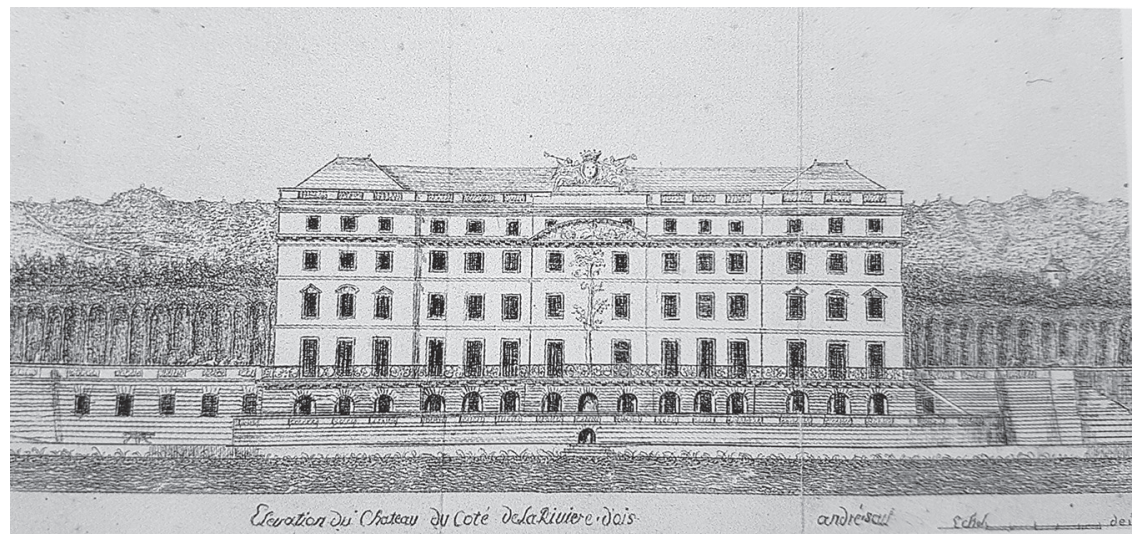

Ill. 3 : Façade donnant du côté des écuries du château de L'Isle-Adam, planche gravée, Jean-Baptiste André, Chronologie historique (...), op. cit., 1809. (C) Cl. de l'auteur.

Mais si le château de L'Isle-Adam est amplement décoré sur ses façades et ses frontons, les écuries princières construites elles aussi par l'architecte Jean-Baptiste André entre 1778 et 1780, afin d'abriter 225 chevaux, comportent également un important décor sculpté dont la paternité revient une nouvelle fois, peut-être en partie à Pierre Mérard, mais surtout à Moitte. Malheureusement, à l'instar du château, ces écuries furent entièrement détruites vers 1810. Ainsi, peu de représentations exactes ayant été conservées de ces écuries considérées comme parmi les plus impressionnantes de leur époque, nous en sommes souvent réduits à des conjonctures quant à l'aspect précis de leur décor sculpté réalisé de 1778 à 1782. Inspirées du spectaculaire décor sculpté des écuries royales de Versailles et des écuries princières de Chantilly appartenant aux cousins Condé, les sculptures de Moitte étaient situées dans le tympan semi-circulaire placé au-dessus des portails principaux en arcades et pourraient avoir été constituées d'un protomé de trois chevaux bondissant dans une allure libre, crinière au vent, représentés en haut-relief. Ce décor sculpté semble avoir été apprécié par les amateurs, car Pahin de la Blancherie, le critiquerédacteur de la revue du Salon de la Correspondance, écrit le 3 juillet 1782 que "la composition est riche, l'exécution pure et grande et les chevaux d'une forme admirable". Certaines estampes anciennes montrent également des oculi entourés de cornes d'abondance ou remplacés par une simple tête de cheval. D'autres tympans de frontons paraissent avoir été classiquement sculptés d'allégories féminines de la Victoire ou de la Renommée encadrant les armoiries des Conti.

\section{Le décor sculpté de la chapelle Conti de l'église Saint-Martin de L'Isle-Adam}

Enfin, un dernier chantier architectural comportant un décor sculpté, s'ouvre au décès de l'avant-dernier prince de Conti. En effet, à la mort de Louis-François de Bourbon en 1776, son fils Louis-François-Joseph, le nouveau prince de Conti, 


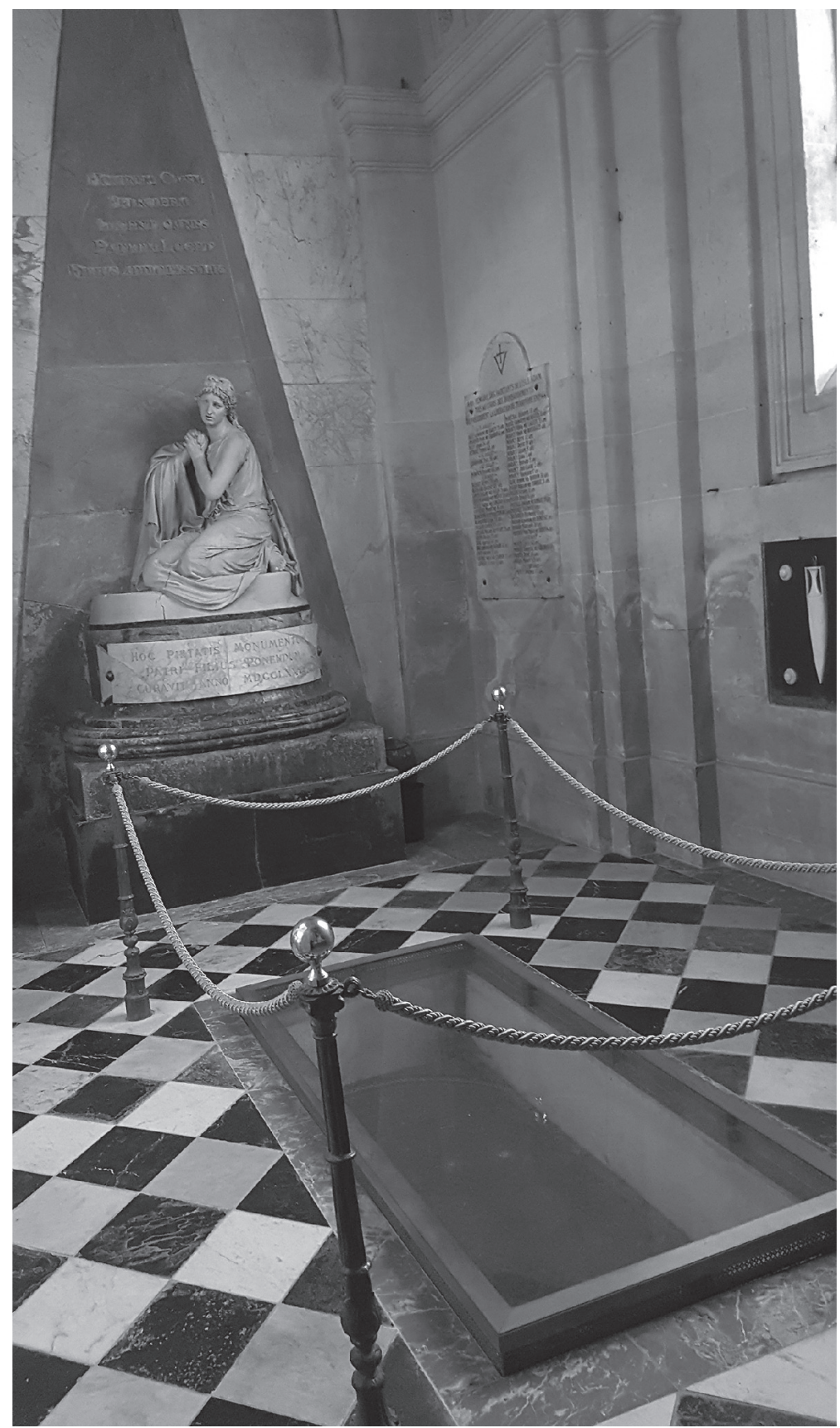

Ill. 4 : L'Isle-Adam, Église Saint-Martin, vue générale actuelle du monument funéraire de Louis-François de Bourbon Conti et de son tombeau. (c) Cl. de l'auteur. 
décide de respecter la volonté de son père ayant souhaité être enterré non pas prestigieusement à Paris, mais modestement dans la petite église Saint-Martin de L'IsleAdam, près de son château et de ses chères forêts giboyeuses où il avait été si heureux. Il ordonne ainsi à son architecte Jean-Marie Morel (1724-1810), qui avait déjà travaillé pour l'aménagement des jardins de son père, la construction d'une chapelle funéraire attenante à la nef, au niveau du transept. Cette chapelle, inaugurée l'année suivante, en 1777 , comportait à droite, un autel en marbre brun Sainte-Anne de Belgique décoré peut-être d'un relief en stuc de Pierre Mérard représentant Saint François d'Assise en prières, couronné des armoiries des Bourbons Conti, tous aujourd'hui disparus, mais surtout, abritait à gauche, le mausolée funéraire du prince décédé (ill. 4).

Ce dernier était composé d'une pyramide de marbre bleu turquin adossé à un mur de marbre blanc et reposant sur un socle ovale, lui aussi en marbre Sainte-Anne de Belgique, le tout accompagné de deux inscriptions archaïsantes en latin, rendant hommage au défunt. Cette disposition générale est à peu près celle existante aujourd'hui. Par contre, était jadis disposé sur ce socle, un génie adolescent en pleurs réalisé en marbre, éteignant de sa main droite une torche de la vie renversée et s'appuyant de la main gauche sur un médaillon de forme ovale en marbre de Carrare, figurant lui, le portrait posthume de profil du défunt, le prince LouisFrançois de Bourbon Conti, décédé l'année précédente, en 1776. L'ensemble était décoré d'ornements ciselés en bronze comme des branches de lauriers ou une balance de la justice, réalisés par des sculpteurs ornemanistes du nom de Jean-Joseph Lemaire et d'un dénommé Bastier.

Le tombeau particulier du prince fut aménagé dans un caveau au centre de la chapelle qui contient encore intact le corps du défunt comme l'a démontré l'ouverture, il y a quelques années, de la plaque funéraire en marbre noir et son inquiétant remplacement actuel par une dalle vitrée éclairée par en dessous, ayant entraîné aussitôt une détérioration rapide de l'une des dernières effigies de princes du sang de l'Ancien régime qui était restée inviolée sous la Révolution.

Le monument funéraire avait été imaginé et exécuté par le sculpteur Pierre Mérard qui ne dut présenter que le simple modèle en plâtre, le jour de l'inauguration, le 2 août 1777. Ce sculpteur un peu oublié aujourd'hui mais qui fut pourtant l'un des plus fins portraitistes de la bonne société de la fin de l'Ancien Régime, semble avoir été auparavant engagé par le prince Louis-François pour travailler à la décoration du château ou des écuries de L'Isle-Adam puisqu'il était pensionné par ce dernier dès 1774. Telle est la raison de son engagement par son fils, le nouveau prince de Conti.

Les chroniqueurs anonymes des célèbres Mémoires secrets publiés sous le nom de Louis Petit de Bachaumont (1690-1771), petit-fils du médecin des Conti, et se rapportant à la vie intellectuelle en France de 1762 et 1787, rapportent que ce modèle du monument Conti fut fort apprécié lors des visites proposées dans l'atelier parisien de Mérard ${ }^{5}$. Démontés dès 1792 et mutilés vers 1794, par les membres

5. Bachaumont, Mémoires secrets pour servir à l'histoire de la République des Lettres en France [...], Londres, éd. John Adamson, 1777-1789, 32 volumes, t. X, p. 344. 
révolutionnaires de la Société populaire de L'Isle-Adam, cette figure du génie et le décor en bronze ont aujourd'hui disparu.

Seul subsiste paradoxalement de l'œuvre de Mérard, le médaillon en marbre $(\mathrm{H} .60 \times \mathrm{L} .30 \mathrm{~cm})$, étrangement retrouvé chez un particulier, avant 1854 dit-on, par Pierre-Charles Dambry (1796-1869), un ancien maire collectionneur et mécène de L'Isle-Adam entre 1834 et 1869, qui l'offre à la paroisse et le réinstalle non pas sur le monument disparu, mais face à l'entrée de la chapelle, sur une longue plaque de marbre noir, placée en contre-bas de la fenêtre. Pourtant, la disposition actuelle du monument funéraire, différente de celle du XVIII ${ }^{\mathrm{e}}$ siècle, présente fort curieusement une statue féminine - ou un très haut-relief - en plâtre de Moitte $(\mathrm{H} .140 \mathrm{~cm}$, non signée ni datée), disposée sous le Premier Empire, en 1811, à l'emplacement du génie à la torche, à une époque où il semble étonnant, d'ailleurs, que les autorités impériales aient pu autoriser la restauration d'un monument funéraire de la famille des Bourbons, alors que le dernier prince de Conti, Louis-François-Joseph, l'ancien commanditaire du monument initial, était lui-même encore vivant, exilé et pensionné à Barcelone jusqu’à sa mort en 1814. Cet arrangement effectué est d'autant plus notable, que cette figure est peut-être le modèle de la statue-relief en marbre de Moitte qui composera le cénotaphe fictif en l'honneur du roi Louis XV, composé en 1841-1842 par l'architecte François Debret (1777-1850), dans la crypte des Bourbons de la cathédrale de Saint-Denis, où elle subsiste encore, même après le démantèlement de ce monument, un peu avant 1881 (statue/haut-relief en marbre, $138 \times 95 \times 60 \mathrm{~cm})$.

Originellement, cette figure agenouillée dans l'attitude de la Douleur, dite aussi de la Charité, aurait été conçue par Moitte (dès 1787 ?) pour un monument funéraire familial qu'il projeta un moment d'ériger en mémoire de son épouse, la peintre Adelaïde-Marie-Anne Castellas (1747-1807), célèbre figure de la Révolution pour avoir conduit en 1789, devant l'Assemblée Nationale, une délégation de femmes d'artistes venues patriotiquement offrir leurs bijoux pour soutenir la République naissante. Exécutée pour un monument qui lui, ne fut pas réalisé pour des raisons encore inconnues, cette statue-relief fut offerte par Moitte - peut-être avec le modèle en plâtre de L'Isle-Adam - sans doute vers 1807, et déposée au musée des Monuments Français d'Alexandre Lenoir, premier musée d'art contemporain de France, où elle fut conservée avant son déplacement en 1818 à Saint-Denis. Cette figure présente des analogies certaines avec d'autres figures funéraires éplorées de cette époque, comme celle de Louis-Claude Vassé pour le tombeau de Paul-Esprit Feydeau de Brou (La Douleur, 1771, marbre, Paris, musée du Louvre).

Le monument Conti de Mérard, sobre, digne et classique, et dont aucune représentation précise n'a encore été identifiée, s’inscrivait en réalité dans une longue suite de tombeaux et de monuments funèbres réalisés selon ce modèle iconographique depuis la fin du XVIII e siècle et surtout sous l'influence de Jean-Antoine Houdon (1741-1828) qui prônait sur ce sujet dès 1774 , un retour à une certaine sévérité antiquisante, comme le mausolée du comte de Vergennes, ministre de la guerre sous Louis XV et décédé en 1787, réalisé par le sculpteur Blaise Barthélémy (1738-1819) et placé en 1818 dans la chapelle des morts de Notre-Dame-de-Versailles. 


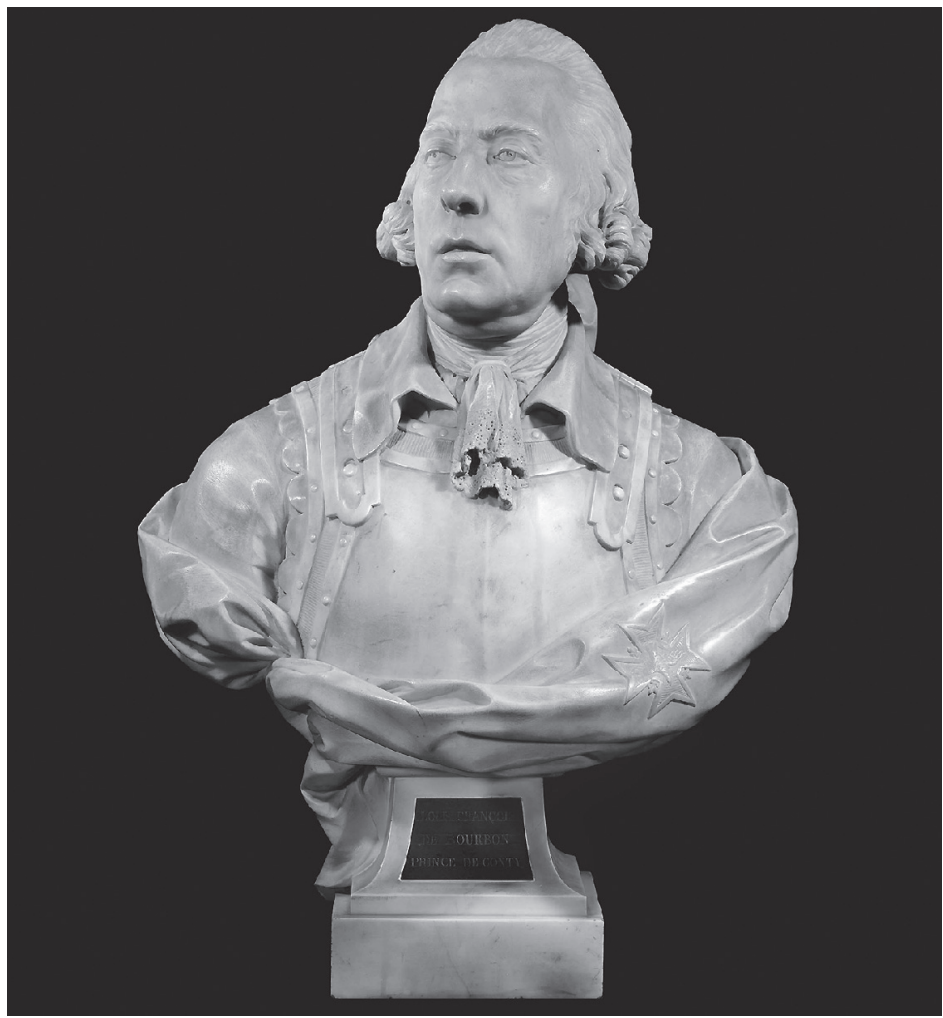

Ill. 5 : Pierre Mérard, Portrait de Louis-François de Bourbon, prince de Conti, 1777, buste en marbre, coll. part. (C) Cl. de l'auteur.

\section{Le portrait en buste de Louis-François de Bourbon Conti}

Au même moment que sont conçus et réalisés ce mausolée et le tombeau de Louis-François de Bourbon Conti par Pierre Mérard, ce dernier finalise également le spectaculaire portrait posthume en buste du prince qui l'avait commandé un peu avant son décès survenu en août 1776 (pour son monument funéraire ?), et dont le modèle original en terre cuite (H. $71,5 \mathrm{~cm}$, Dijon, MBA), daté d'octobre 1776, précède la version définitive en marbre datée de mai 1777 , et vendue récemment par l'actuel duc de Rohan, descendant de Louis-Antoine-Auguste de Rohan, comte de Chabot (1733-1807), l'un des familiers du prince Louis-François (buste en marbre, H. $62 \mathrm{~cm}$, vente à Paris, chez Christie's, 6 novembre 2014, no 430). D'un réalisme impressionnant, ce portrait traduit avec justesse la complexe personnalité, tout à la fois hautaine et désabusée, de ce prince du sang qui aima tant l'art de la sculpture (ill. 5).

Frédéric CHAPPEY

Maître de conférences en histoire de l'art contemporain à l'université de Lille 